\title{
An outbreak of hepatitis A associated with a contaminated well in a middle school, Guangxi, China
}

\author{
Xu Ye-qing, ab Cui Fu-qing, ${ }^{c}$ Zhuo Jia-tong, ${ }^{d}$ Zhang Guo-ming, ${ }^{c}$ Du Jin-fa, ${ }^{d}$ Den Qu-yun ${ }^{c}$ and Luo Hui-min ${ }^{c}$ \\ Correspondence to: Luo Hui-ming (e-mail:Hmluo@vip.sina.com).
}

Background: In May 2012, an outbreak of viral hepatitis A was reported to the Guangxi Center for Disease Control and Prevention from a middle school in Liujiang County. An investigation was conducted to identify the cause and mode of transmission and to recommend control and prevention measures.

Methods: A case was defined as any person from the middle school with onset of fatigue, anorexia, abdominal pain, diarrhoea or jaundice from 20 February to 20 May 2012. We compared attack rates (AR) between boys and girls, assuming that only boys used well water and girls used pipeline water. We then selected 133 students from three classes in each of the three grades to compare AR by reported water source and drinking history.

Results: There were 22 cases, an AR of 3.8\% (21/553) for students and $1.5 \%$ for teachers (1/65). Those who used well water were $8.7(95 \%$ confidence interval $[\mathrm{Cl}]=2.1-37.2)$ times more likely to be ill than those using pipeline water. The cohort study showed that students who reported using well water daily were $5.2(95 \% \mathrm{Cl}=0.7-41.8)$ times more likely to be ill than those that reported using the pipeline water daily. Eighteen cases were confirmed as hepatitis $A$.

Conclusion: This hepatitis A outbreak was potentially caused by a contaminated school well. We recommended that the school discontinue using the well and that the students should drink boiled water. As there is a vaccine for hepatitis $A$, we recommended that several doses of the vaccine be stored for controlling outbreaks and for immunizing susceptible populations in future outbreaks.

$\mathrm{H}$ epatitis A infection is an acute, usually selflimiting disease of the liver caused by hepatitis A virus. Hepatitis A virus can be transmitted through food, water and from person to person, primarily by the faecal-oral route. Risk factors for hepatitis $A$ virus infection are related to resistance of hepatitis $A$ virus to the environment and include poor sanitation in large areas of the world and abundant hepatitis A virus shedding in faeces. Under these conditions epidemics can arise from water and food contamination. ${ }^{1-3}$ The incidence of hepatitis $A$ is closely related to socioeconomic development. ${ }^{4}$ An estimated 1.5 million clinical cases of hepatitis A occur each year worldwide. ${ }^{1}$ In areas where Hepatitis A virus is highly endemic, most hepatitis A virus infections occur during early childhood. ${ }^{1}$

Viral hepatitis $A$ is a category $B$ infectious disease in China; therefore, all cases of hepatitis $A$ are reported by hospitals to the Chinese Center for Disease Control and Prevention (CDC). Though incidence is low, hepatitis
A can cause large outbreaks resulting in huge economic loss and social panic ${ }^{5}$ such as the large outbreak of about 310000 people in Shanghai in $1988 .{ }^{2}$ Hepatitis A vaccine was included in routine immunization in China in 2008, with children aged 18 months eligible for a free injection of hepatitis $A$ vaccine.

In May 2012, an outbreak of viral hepatitis A was reported to the Guangxi CDC from a middle school in a rural location in Liujiang County. Fellows from the China Field Epidemiology Training Programme, along with Guangxi CDC staff, conducted an investigation to identify the cause and mode of transmission and to recommend control and prevention measures.

\section{METHODS}

We conducted an outbreak investigation including a retrospective cohort study. A case was defined as any

\footnotetext{
Chinese Field Epidemiology Training Programme, Beijing, China.

Xinjiang Uygur Autonomous Region Center for Disease Control and Prevention, Urumqi, China

National Immunization Programme, Chinese Center for Disease Control and Prevention, Beijing, China.

Guangxi Zhuangzu Autonomous Region Center for Disease Control and Prevention, Guangxi, China.

Submitted: 28 October 2012; Published: 20 December 2012

doi: 10.5365/wpsar.2012.3.4.014
} 
person from the school with onset of fatigue, anorexia, abdominal pain, diarrhoea or jaundice from 20 February to 20 May 2012, with all other diagnoses excluded. Active case finding was conducted by reviewing the county hospital's medical registrations and by interviewing students and teachers from the school. We used a structured questionnaire, which included questions on water exposures and other environmental factors. Attack rates (AR) were calculated using the number of teachers and students in the school as denominators. We found there were no vaccination records for the local area hepatitis A vaccination programme before 2007.

To verify the hypothesis that well water was the cause of this hepatitis A outbreak, we conducted a retrospective cohort study. We selected 133 students from three classes in the three grades. We used a more specific questionnaire that focused on drinking water. We also evaluated whether the students drank boiled water and the frequency. Those who answered "seldom" were scored 1, "sometimes" were scored 2, "often" were scored 3, and "always" were scored 4. The scores were analysed using logistic regression using Epi Info.

We conducted an environmental investigation at the school to determine food and water supply arrangements. The visit occurred after the implicated well was closed, so no water samples were able to be collected. Serum samples were collected from student and teacher cases between 20 February and 20 May 2012 and tested using the hepatitis A virus immunoglobulin test.

\section{RESULTS}

\section{Environmental investigation}

The environmental investigation found that all students lived on the school campus. The middle school comprised three grades of 12 classes with 553 students (288 boys and 265 girls) and 65 teachers.

There were two water sources at the school, one from the waterworks which was piped into the school buildings, the other from a well obtained from outside taps. The pipeline water was treated whereas the well water was not treated. The well was located close to potential environmental contamination sources, including a vegetable garden often fertilized with toilet faeces. There were no water facilities or toilets in the boys' dormitory, so the boys used the outside well water for bathing and mouth wash, with some even drinking the tap water directly. There were water facilities and toilets in the girls' dormitory, so they used the pipeline water for daily washing. The teachers' dormitory also used pipeline water. However, the teacher case lived in a domestic cottage next to the school well and used the well water for cooking and washing.

The school had one canteen that provided three meals each day. It used pipeline water for daily food preparation and for drinking water. As only one teacher and two female students were ill, compared with 19 male students, a foodborne outbreak of hepatitis was considered unlikely. Also, the number of cases declined after use of the well water was stopped.

There were no hepatitis A cases reported among other people in this county.

\section{Cases}

There were 22 cases whose clinical symptoms consisted of malaise $(100 \%)$, anorexia $(100 \%)$, nausea $(72 \%)$, jaundice $(41 \%)$, abdominal pain $(41 \%)$, fever $(32 \%)$ and vomiting (27\%). There were 21 student cases giving an AR of 3.8\% (21/553) and one teacher case giving an AR of $1.5 \%(1 / 65)$. There were no severe cases or deaths. Of the 22 cases, 18 were laboratory confirmed as hepatitis A virus.

The epidemic curve showed the first case occurred on 11 April, and that cases continued for the next 30 days (Figure 1 ).

The teacher case was 54 years old; the student cases ranged in age from 13 to 16 years. Most of the cases were male students $(n=19)$ with an AR of $6.6 \%$ (19/288). The two female student cases corresponded to an AR of $0.8 \%(2 / 265)$. Assuming that all male students used the well water and all female students used pipeline water, those that used the well water were $8.7(95 \%$ confidence interval $[\mathrm{Cl}]=2.1-37.2)$ times more likely to be ill.

\section{Cohort study}

In the cohort study of 133 students, the AR of those who reported using the school well water daily was $10.4 \%$ (7/67) compared to $1.8 \%(1 / 56)$ of those who reported using pipeline water $(R R=5.2,95 \% \mathrm{Cl}=0.7-41.8)$. 
Figure 1. The epidemic curve for outbreak cases in a middle school, Guangxi Province, China, 2012

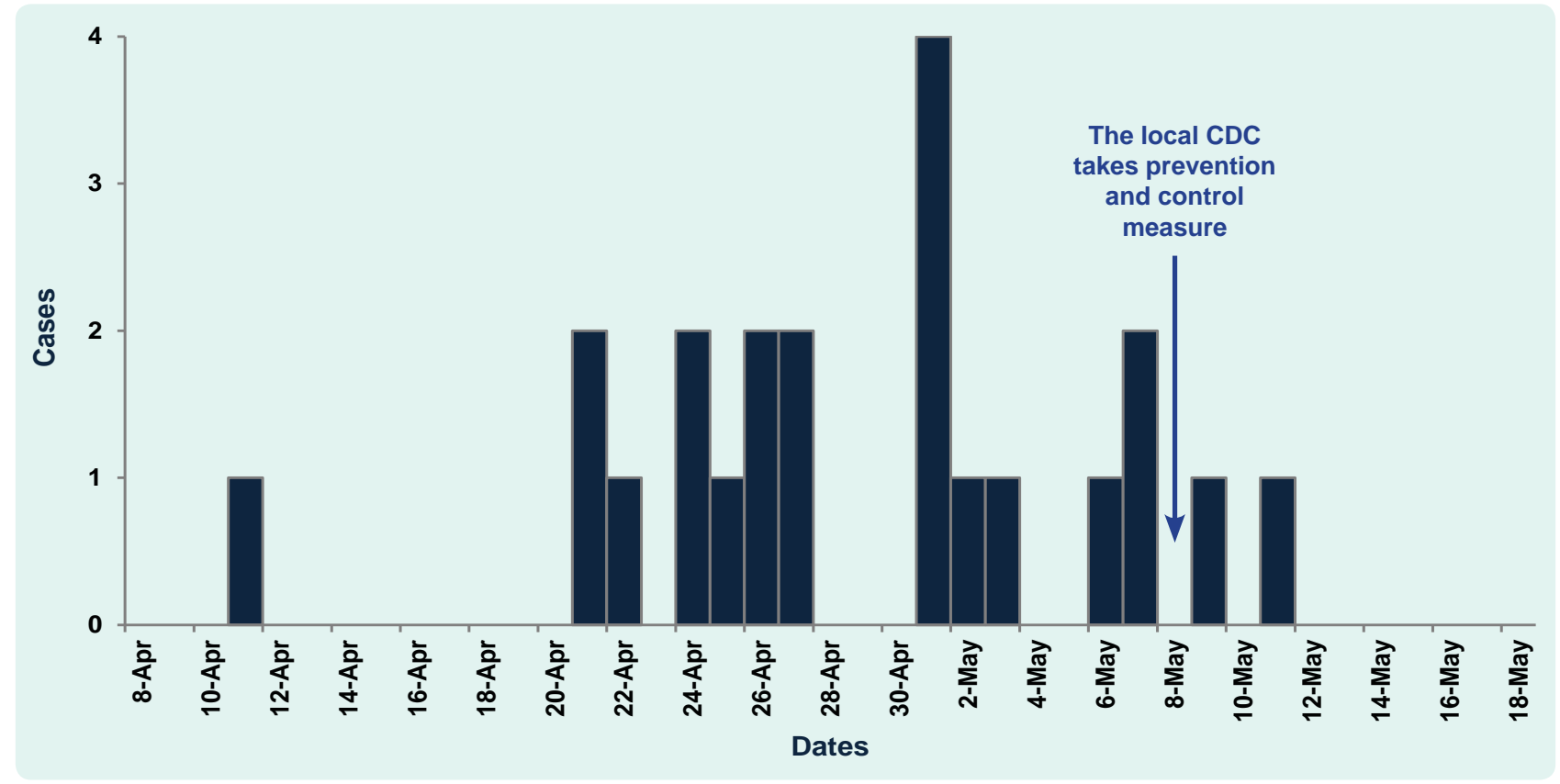

Logistic regression analysis of drinking boiled water showed that this increased, the risk for morbidity decreased by $15 \%$ (OR: $0.85,95 \% \mathrm{Cl}=0.48-1.5)$.

\section{DISCUSSION}

This hepatitis A outbreak in a middle school in Guangxi province, China, was most likely caused by contaminated well water. There were 22 cases with an AR of $3.6 \% ; 19$ cases were male students. Being a male student was associated with illness, because they used the well water, whereas the females at the school had access to and used treated pipeline water. The well water was delivered to boys for daily washing, and some students also drank the well water directly. The results of the cohort study also showed that students who reported using the well water were associated with illness. The well water was not treated and was located next to a vegetable field that was often fertilized by faeces, a possible source of contamination.

There was one teacher case and only two female student cases. As all students and teachers eat at the same cafeteria at the school, the likeliness of the outbreak being caused by food is very small. No additional hepatitis A cases were reported among people living near this school, discounting community transmission.
Hepatitis A is transmitted by contaminated water and food including seafood such as blood clams. It can cause both sporadic cases and outbreaks. Since 1990 in China, the reported number and morbidity of hepatitis A has been decreasing, although there is a significant difference among the provinces, with the less well-off regions having higher morbidity. ${ }^{5}$ Outbreaks mainly happen in schools due to the concentration of susceptible population, intensive living, daily diet and drinking conditions. ${ }^{6}$

This outbreak of hepatitis A revealed the management issue of using wells in rural schools. Due to the economy, quite a few schools that cannot afford pipeline water fees use well water instead. Water drawn from wells located in low-lying places can become contaminated after rain. This can result in outbreaks of intestinal infectious diseases such as this hepatitis A outbreak. Although the well water in this outbreak was for domestic use, some students drank the water directly.

There are some limitations to our investigation. We were only able to administer the cohort study questionnaires to 133 students, which comprised only eight ill students. We were also unable to test the well water as the well had been closed before our arrival at the 
school. However, despite these limitations, it is plausible that contaminated well water caused this outbreak.

As a result of this outbreak, we recommended that the school discontinue using the well and that the students drink boiled water. We also recommended that drinking water safety for rural school residents be investigated through close collaboration among the government, water conservancy and health and education bureaux. This could potentially prevent future outbreaks. We also recommended that hepatitis $A$ vaccines be stored for use in controlling outbreak through immunizing susceptible populations. ${ }^{7,8}$

\section{Conflicts of interest}

None declared.

\section{Funding}

None.

\section{References:}

1. Fiore AE, Wasley A, Bell BP. Prevention of hepatitis A through active or passive immunization. MMWR. Morbidity and Mortality Weekly Report, 2006, 55 RR07:1-23. pmid:16708058

2. Wu J et al. Spring 1988 Shanghai hepatitis a outbreak indirect economic loss analysis. Chinese Public Health Management, 1990, 4:41-43.

3. Lemon SM. Type A viral hepatitis: epidemiology, diagnosis, and prevention. Clinical Chemistry, 1997, 43(8[B]):14941499.

4. Centers for Disease Control and Prevention (CDC). Prevention of hepatitis $A$ through active or passive immunization: recommendations of the Advisory Committee on Immunization Practices. MMWR. Morbidity and Mortality Weekly Report, 1996, 45(RR15):1-30. pmid:9005304

5. Zheng $\mathrm{H}$ et al. Epidemiological analysis on hepatitis $\mathrm{A}$ in China during 2004-2006. Chinese Journal of Vaccines and Immunization, 2007, 13:336-341.

6. Li $\mathrm{LI}$ et al. Analysis and countermeasure for current situation of hepatitis A, B in China. Disease Surveillance, 2009, 24:307312.

7. Hai-tian SUI et al. Epidemic characteristics on hepatitis $A$ in China during 1990-2006. Chinese Journal of Vaccines and Immunization, 2007, 13:466-469.

8. Committee on Infectious Diseases. Hepatitis A vaccine recommendations. Pediatric, 2007, 12:189-199. 